\title{
Phthisis Bulbi and Leptomeningeal Metastasis in a Case of Trilateral Retinoblastoma
}

\author{
Gupta MK ${ }^{1}$, Rauniyar RK ${ }^{1}$, Ahmad $K^{1}$, Pant AR ${ }^{1}$, Kumar $A^{1}$, Shah $S^{2}$ \\ ${ }^{1}$ Department of Radiodiagnosis and Imaging \& ${ }^{2}$ Department of Ophthalmology, B.P. Koirala \\ Institute of Health Sciences, Dharan, Nepal
}

\begin{abstract}
Retinoblastoma (RB) is the most common ocular malignant neoplasm of childhood. Bilateral or unilateral intraocular RB in conjunction with a primitive neuroectodermal tumor (PNET) of the pineal region (i.e. pineoblastoma) is termed as trilateral retinoblastoma (TRB). Retinoblastoma may spread along the optic nerve to the intracranial compartment to give drop metastases in the subarachnoid space or it can spread through hematogenous and lymphatic routes. Phthisis bulbi (PB) is a relatively uncommon presentation of retinoblastoma. We report a case of trilateral retinoblastoma with phthisical left eye and extensive leptomeningeal metastasis in a 3-year-old female child.
\end{abstract}

Keywords: Leptomeningeal metastasis, Phthisis bulbi, Pineoblastoma, PNET, Trilateral retinoblastoma.

\section{Introduction}

$\mathrm{RB}$ is the most common intraocular malignant tumor of early childhood, occurring in $1 / 17,000$ live births. ${ }^{1}$ Retinoblastoma cells contain a mutation or deletion of the retinoblastoma gene (RB1 gene), a tumor-suppressor gene, located on chromosome 13q14. ${ }^{2,3}$ About $40 \%$ of patients with retinoblastoma are reported to have hereditary disease (usually bilateral), while the remainder $(60 \%)$ have unilateral

Corrospondence to: Dr. Mukesh Kumar Gupta, Associate Professor, Department of Radiodiagnosis and Imaging, B.P. Koirala Institute of Health Sciences, Dharan, Nepal. E-mail: mukeshgupta148@yahoo.com nonhereditary disease. A midline intracranial PNET in suprasellar or the pineal region (pineoblastoma) is reported in association with hereditary retinoblastoma in about 5$15 \%$ of patients referred as trilateral retinoblastoma. ${ }^{4}$ Phthisis bulbi is a rare presentation of retinoblastoma with approximately $2 \%$ incidence. The cause of $\mathrm{PB}$ is thought to be due to occlusion of the central retinal vessels, which may lead to partial or total regression of the intraocular tumor. ${ }^{5}$ CSF seeding by RB presents with diffuse leptomeningeal enhancement in the subarachnoid and intrathecal spaces. ${ }^{6} \mathrm{We}$ report an unusual case of phthisis bulbi in a 3-year-old female child with trilateral retinoblastoma with extensive leptomeningeal spread of the disease. 


\section{Case Report}

A 3-year-old female child presented with bilateral leukocoria, small left eye and inability to see the objects. Family history, past ocular history and medical history were unremarkable. Ocular examination revealed bilateral dense vitreous mass obscuring the posterior view, phthisical left eye and inability to follow the light with either of the eye.

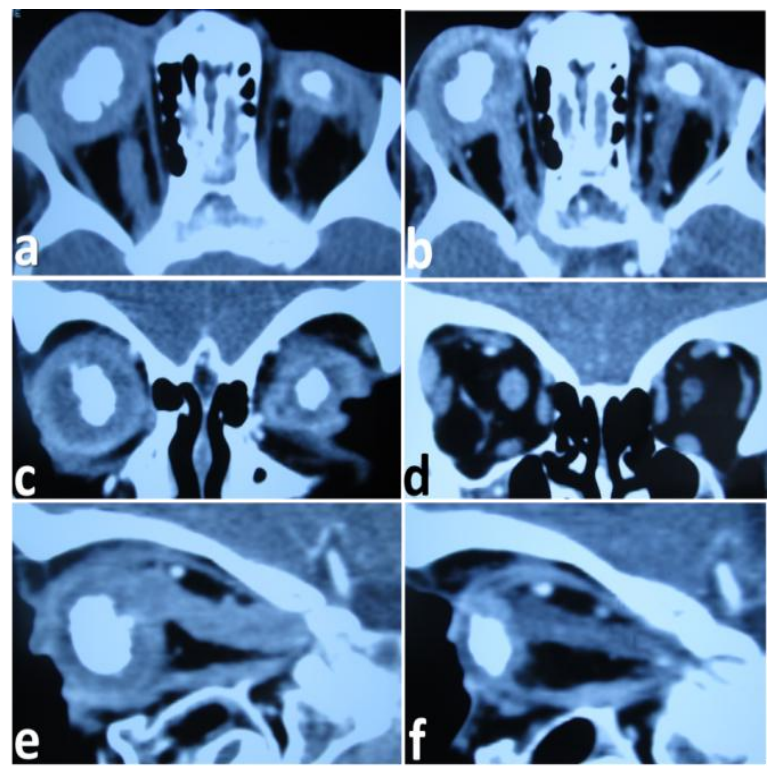

Fig. 1: NCCT axial (a) and CECT axial (b), coronal (c, d), sagittal (e: right, f: left) images of the orbit shows bilateral mildly enhancing heterogeneous intraocular calcified mass occupying almost whole of the globe with markedly thickened bilateral optic nerves, suggesting bilateral retinoblastoma infiltrating the optic nerves. The left globe is small, deformed and shrunken suggesting phthisis bulbi.

Ultrasonography showed bilateral intraocular solid mass with coarse calcification occupying almost whole of the globes with phthisical left eye. CT scan of the orbit (Fig.1) showed bilateral mildly enhancing heterogeneous intraocular calcified mass occupying almost whole of the globe with markedly thickened bilateral optic nerves, suggesting bilateral retinoblastoma infiltrating the optic nerves. In addition, the left globe was small, deformed and shrunken suggesting phthisis bulbi. CT scan of the brain (Fig.2) revealed a large lobulated mildly enhancing heterogeneous mass of size $8 \times 6.5 \times 6 \mathrm{~cm}$ with multiple coarse calcifications in the pineal region extending into the right parieto-occipital lobe and causing displacement $\&$ compression of $3^{\text {rd }}$ ventricle with dilated bilateral lateral ventricles. This intracranial mass was suggestive of pineoblastoma occurring in association with bilateral retinoblastoma i.e. synchronous trilateral retinoblastoma. No suprasellar mass was identified. In addition, there was wide spread marked thickening and nodular enhancement of the leptomeninges in the basal cisterns suggesting leptomeningeal spread of the disease. The child was referred to the oncology center for treatment.

\section{Discussion}

Retinoblastoma is a highly malignant tumor of the developing retina usually seen before the age of 5 years, and may be unilateral, bilateral, trilateral or quadrilateral. Trilateral retinoblastoma is a well-known syndrome which consists of unilateral or bilateral RB associated with an intracranial neuroblastic tumor. ${ }^{4}$ The overall reported incidence of trilateral $\mathrm{RB}$ in patients with unilateral or bilateral $\mathrm{RB}$ is $1.5-5 \%$, but it is higher in children with bilateral RB (2-11\%). Although the associated primary intracranial tumor in patients with trilateral RB is most frequently located in the pineal region, some tumors occur in the suprasellar or parasellar region. ${ }^{8}$ Quadrilateral $\mathrm{RB}$ refers to the occurrence of bilateral ocular RB and 
primary intracranial PNETs in both suprasellar and pineal regions. ${ }^{6}$ These midline intracranial PNETs may be discovered after several years of detection of ocular tumor. In a study by Paulino, the median time intervals from the diagnosis of $\mathrm{RB}$ to the development of a pineal and suprasellar tumor were 24 months and 1 month respectively. ${ }^{9}$ In a study on 93 patients by Kivela, intracranial tumors were found before $\mathrm{RB}$, concomitantly with $\mathrm{RB}$ and after $\mathrm{RB}$ in $3(3 \%), 13(14 \%)$ and 77 $(83 \%)$ cases respectively. ${ }^{4}$

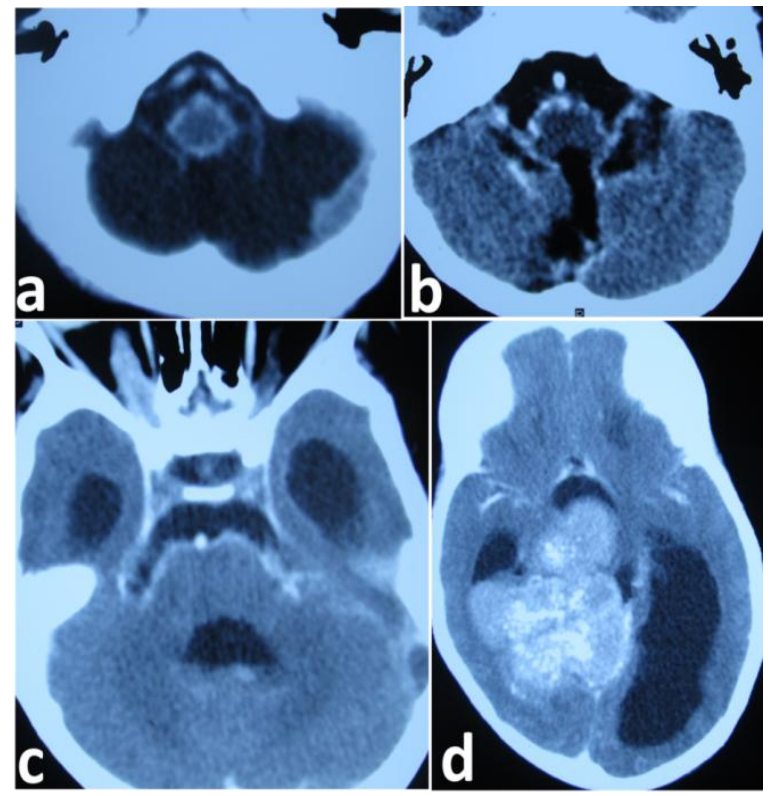

Fig.2: CECT of the brain shows a large mildly enhancing heterogeneous mass (fig. d) with coarse calcifications in the pineal region extending into the right parietooccipital lobe causing displacement and compression of $3^{\text {rd }}$ ventricle with dilated bilateral lateral ventricles suggestive of pineoblastoma occurring in association with bilateral retinoblastoma i.e. synchronous trilateral retinoblastoma. There is wide spread marked thickening and nodular enhancement of the leptomeninges in the basal cisterns (fig. a, b \& c) suggesting leptomeningeal spread of the disease.
Both the tumors (i.e. bilateral ocular RB and pineoblastoma) were found at the same time in our case.The three major causes of death in patients with $\mathrm{RB}$ are metastases, associated second non-ocular cancers and trilateral RB. The CNS, bone and regional lymph nodes are common sites for metastasis in RB. ${ }^{10}$ Retinoblastoma may spread along the optic nerve to the intracranial compartment to give drop metastases in the subarachnoid space, in which tumor cells infiltrate the subarachnoid space of the optic nerve sheath and disseminate into the CSF of the central nervous system. Less commonly, leptomeningeal spread may occur from a primary intracranial tumor in patients with trilateral RB. ${ }^{11}$

Phthisis bulbi is a rare presentation of $\mathrm{RB}$, especially in advanced cases, which often occurs after an ocular inflammatory episode possibly related to intraocular tumor infarction due to occlusion of central retinal vessel. ${ }^{5}$ Mullaney et $\mathrm{al}^{5}$ found that $2.7 \%$ of patients had RB coincident with phthisis bulbi and all enucleated phthisical globes had residual viable tumor cells.

Diagnosis and treatment of RB are based on multidisciplinary approach. Radiological imagings play important role in diagnosis, staging, treatment monitoring and follow-up of RB. With the increasing trends toward preserving the useful vision, especially in cases of bilateral RB or suspected intracranial involvement, the role of radiological imagings has been tremendously increased in recent era. CT scan detects intraocular, extraocular, and intracranial disease extent with excellent delineation of bony details and depiction of tumoral calcifications. CT scan is the mainstay of the 
radiological diagnosis, primarily because of its high sensitivity to detect calcification which is an important feature of RB. Retinoblastoma is seen on CT as an intraocular mass, which is calcified in about 95\% of cases, located posterior to the lens and shows mild or moderate enhancement. ${ }^{12}$ In advanced cases the tumor may fill the entire globe. The diagnosis of RB is highly likely when intraocular calcifications are present in a child less than 3 years of age.

The prognosis of trilateral $\mathrm{RB}$ is very poor and majority of the patients die of disseminated neuraxis disease in less than 9 months. Rare survivors of trilateral RB are those diagnosed early by screening imaging and treated with intensive chemotherapy with or without craniospinal irradiation. ${ }^{4,13}$ Because of very poor prognosis of trilateral $\mathrm{RB}$, screening neuroimaging has become a routine practice now a days for early detection of asymptomatic intracranial tumors. Although there is wide spread variations in the screening programs for detecting trilateral $\mathrm{RB}$, it seems rational to screen every 3 months during first year and at least twice a year for the next 3 years after diagnosis of RB. It is advisable to use MRI for screening neuroimaging to avoid radiation exposure and reduce the risk of associated second cancers. MRI is also an efficient method to detect drop metastasis in the CNS. ${ }^{4}$

\section{Conclusion}

Retinoblastoma is a highly malignant tumor of the developing retina usually seen before 5 years of age. Trilateral RB refers to a midline intracranial PNET occurring in association with unilateral or bilateral retinoblastoma and has a very poor prognosis. Phthisis bulbi is a rare presentation of retinoblastoma, especially in advanced cases, with about $2 \%$ incidence. $\mathrm{RB}$ may disseminate into the CSF of the CNS through subarachnoid space of the optic nerve sheath resulting in leptomeningeal spread of the disease.

\section{References}

1. Moll AC, Kuik DJ, Bouter LM, Den OW, Bezemer PD, Koten JW, et al. Incidence and survival of retinoblastoma in The Netherlands: a register based study 18621995. Br J Ophthalmol 1997;81(7):55962.

2. Friend SH, Bernards R, Rogelj $S$, Weinberg RA, Rapaport JM, Albert DM, et al. A human DNA segment with properties of the gene that predisposes to retinoblastoma and osteosarcoma. Nature 1986;323(6089):643-46.

3. Ganesh A, Kenue RK, Mitra S. Retinoblastoma and the $13 \mathrm{q}$ deletion syndrome. J Pediatr Ophthalmol Strabismus 2001;38(4):247-50.

4. Kivela T. Trilateral retinoblastoma: a meta-analysis of hereditary retinoblastoma associated with primary ectopic intracranial retinoblastoma. J Clin Oncol 1999;17(6):1829-37.

5. Mullaney PB, Karcioglu ZA, Al-mesfer S, Abboud EB. Presentation of retinoblastoma as phthisis bulbi. Eye 1997;11:403-408.

6. Razek AA, Elkhamary S. MRI of retinoblastoma. $\mathrm{Br} \quad \mathrm{J} \quad$ Radiol 2011;84(1005):775-784. 
7. Blach LE, McCormick B, Abramson DH, Ellsworth RM. Trilateral retinoblastomaincidence and outcome: a decade of experience. Int J Radiat Oncol Biol Phys 1994;29(4):729-733.

8. De Potter P, Shields CL, Shields JA. Clinical variations of trilateral retinoblastoma: a report of 13 cases. J Pediatr Ophthalmol Strabismus 1994;31(1):26-31.

9. Paulino AC. Trilateral retinoblastoma: is the location of the intracranial tumor important? Cancer 1999;86(1):135-41.

10. Gunduz K, Sheilds CL. Retinoblastoma update. American Academy of Ophthalmology 2005;23(7):1-18.
11. Rauschecker AM, Patel CV, Yeom KW, Eisenhut CA, Gawande RS, O'Brien JM, et al. High-resolution MR imaging of the orbit in patients with retinoblastoma. Radiographics 2012;32(5):1307-26.

12. Lindahl S. Computed tomography of retinoblastoma. Acta Radiol Diagn 1986;27(5):513-518.

13. Dunkel IJ, Jubran RF, Gururangan S, Chantada GL, Finlay JL, Goldman S, et al. Trilateral retinoblastoma: potentially curable with intensive chemotherapy. Pediatr Blood Cancer 2010;54(3):384-7. 